\title{
TORSIÓN ANEXIAL Y EMBARAZO: RESOLUCIÓN LAPAROSCÓPI- CA MÍNIMAMENTE INVASIVA, CON ENTRADA BAJO VISIÓN DIRECTA
}

\author{
José Luis Troncoso J. ${ }^{1}$, Paolo Ricci A. ${ }^{1}$, Ricardo Susaeta C. ${ }^{2}$, Juan Carlos \\ Devoto G. ${ }^{3}$
}

${ }^{1}$ Departamento de Ginecología y Obstetricia, ${ }^{2}$ Departamento de Urología, ${ }^{3}$ Departamento de Anestesiología, Clínica Las Condes.

\section{RESUMEN}

Las masas anexiales durante el embarazo son de baja frecuencia, sin embargo tienen una alta probabilidad de complicarse, produciéndose torsión hasta en un $25 \%$ de ellas. Ante la necesidad de una resolución quirúrgica, debemos tener en cuanta los riesgos de desencadenar una pérdida fetal, como resultado de una cirugía muy invasiva. Gracias a los avances tecnológicos, el mayor conocimiento y manejo de anestesia durante el embarazo, la laparoscopia debería constituir la mejor vía para realizar estas cirugías. De esta forma, se aplican todos los principios de mínima invasión, facilitando un menor daño y rápida recuperación, con poca probabilidad de afectar el embarazo. Presentamos un caso de quiste anexial complicado con torsión, durante el segundo trimestre del embarazo, resuelto por medio de cirugía laparoscópica, con entrada bajo visión directa con cámara, para reducir la posibilidad de daño uterino.

PALABRAS CLAVE: Embarazo y cirugía, quiste anexial complicado, laparoscopia, cirugía mínimamente invasiva

\section{SUMMARY}

The adnexal masses during pregnancy are of low frequency; nevertheless they have a high probability of complication. The torsion can be observed in a $25 \%$ of them. When they need surgery, we must be aware of the risks and morbidity imposed to the fetus and the patient. The technological advance and best knowledge of fetal physiology and hemodynamics plus anesthesia during the pregnancy, allow that laparoscopy constitutes the best via to perform this surgery. By the way, all principles of minor invasion are applied, with short hospital stay and lesser morbidity affecting the pregnancy. We present a case of a complicated adnexal cyst, with torsion, during the second trimester of the pregnancy. A laparoscopic surgery was performed, with entrance under direct camera vision, to reduce uterine damage.

KEY WORDS: Pregnancy and surgery, complicated adnexal cyst, laparoscopy, minimally invasive surgery 


\section{INTRODUCCIÓN}

Tradicionalmente se ha considerado el embarazo intrauterino como una contraindicación relativa para la cirugía laparoscópica. Actualmente existen numerosas publicaciones que muestran la seguridad y beneficios de esta vía, cuando se requiere una solución quirúrgica a patología abdominal aguda o patología anexial de urgencia, que complica a una paciente embarazada (1-3).

La laparoscopia ha demostrado ventajas frente a la cirugía realizada por laparotomía, al tratarse de una técnica de menor invasión $(4,5)$. Lo anterior puede beneficiar a la embarazada y el feto. Entre las causas que pueden motivar una cirugía de urgencia por laparoscopía, se encuentran la apendicitis aguda (6), la colecistitis aguda (7) y patología anexial complicada (8-10).

Los tumores anexiales pueden afectar entre el 0,01 y $0,06 \%$ de los embarazos (11-14). Se estima que el $2,9 \%$ de los tumores pueden ser malignos o de baja malignidad (borderline) (15).

Sin embargo, los tumores benignos, y sobretodo, los de mayor tamaño, pueden complicarse por la torsión o ruptura. La torsión es la complicación más frecuente de las masas anexiales, afectando hasta un $25 \%$ de los casos (16).

A continuación presentamos un caso de torsión de quiste anexial, durante el segundo trimestre de embarazo, con resolución por vía laparoscópica.

\section{Caso Clínico}

Paciente de 23 años, portadora de su tercer embarazo, con antecedente de dos abortos previos. Cursa al momento del ingreso con una edad gestacional de 23 semanas, controlada periódicamente en otro centro de salud. Ingresa al Servicio de Urgencia Clínica Las Condes por dolor tipo cólico, localizado en flanco izquierdo, con irradiación hacia la región lumbar e inguinal izquierda. Este dolor tenía siete días de evolución, que hasta horas previas al ingreso disminuía con el reposo, para luego aumentar en intensidad progresivamente y no ceder con ninguna medida, pero siempre con características de dolor tipo cólico.

Al ingreso fue evaluada por urólogo, al plantearse el diagnóstico diferencial de cólico ureteral izquierdo. La paciente se encontraba estable, sin compromiso hemodinámico, ni signos de abdomen agudo, con unidad feto-placentaria indemne.

Entre los exámenes de ingreso, la orina completa y el hemograma eran normales y PCR de 2 $\mathrm{mg} / \mathrm{L}$. La ecotomografía abdominal resultó dentro de límites normales.
La ecografía fetal demostró que cursaba con una gestación normal, de 673 gramos, con biometría concordante a la amenorrea. A nivel anexial izquierdo se observó una estructura de aspecto quística, adyacente al ovario, que medía 6,5 x 4,5 $\mathrm{cm}$. El ovario izquierdo se identificó de estructura y tamaño normal y el doppler demostró que presentaba un flujo normal. Toda esta zona era muy sensible a la exploración con el transductor de pared abdominal.

El dolor evolucionó hasta hacerse más constante, sin carácter cólico. Se utilizó ketorolaco $30 \mathrm{mg}$ por vía endovenosa, cediendo parcialmente el dolor mientras duraba el efecto del medicamento.

Bajo el diagnóstico presuntivo de lesión anexial izquierda complicada, con una probable torsión en curso, se procedió a informarle a la paciente y sus familiares las posibles alternativas terapéuticas.

Una nueva evaluación de los parámetros de laboratorio con hemograma-VHS, PCR y orina completa, no demostraron variación respecto a los realizados al ingreso. Tampoco hubo variación en una nueva ecotomografía obstétrica con doppler anexial.

El dolor abdominal persistía, hasta hacerse más tarde resistente a la terapia con el analgésico endovenoso, por lo que se agregaban más elementos para sospechar que nos encontrábamos frente a una torsión anexial complicada. Por este motivo, se decidió realizar una cirugía por vía laparoscópica, previa discusión en equipo, incluyendo anestesiólogo y con consentimiento informado de la paciente y sus familiares directos.

Se colocó a la paciente en decúbito dorsal, con una ligera lateralización a izquierda y Trendelemburg moderado. Se instaló sonda Foley. Bajo anestesia general, se introdujo la aguja de Veress por la zona umbilical, utilizando CO2 con flujo de 2 litros por minuto y presión de $15 \mathrm{mmHg}$. Luego se introdujo el primer trocar a través de la zona umbilical. Para ello se utilizó el trocar Xcel (Endopath Xcel, Ethicon, Inc) que es blindado, de $12 \mathrm{~mm}$, transparente y que tiene la característica de permitir colocar la cámara endoscópica en su interior (Figura 1). De tal manera, con muy poca distorsión, se realizó entrada en la pared abdominal, observando los diferentes planos, traspasando el peritoneo parietal hasta la cavidad abdominal. La inserción del trocar se detuvo al visualizar la pared uterina (Figura 1). Así se redujo la probabilidad de daño uterino durante la entrada. Se utilizaron dos trocares accesorios de $5 \mathrm{~mm}$, con la misma punta del principal. Uno se localizó a nivel suprapúbico y el otro parailíaco izquierdo alto. 


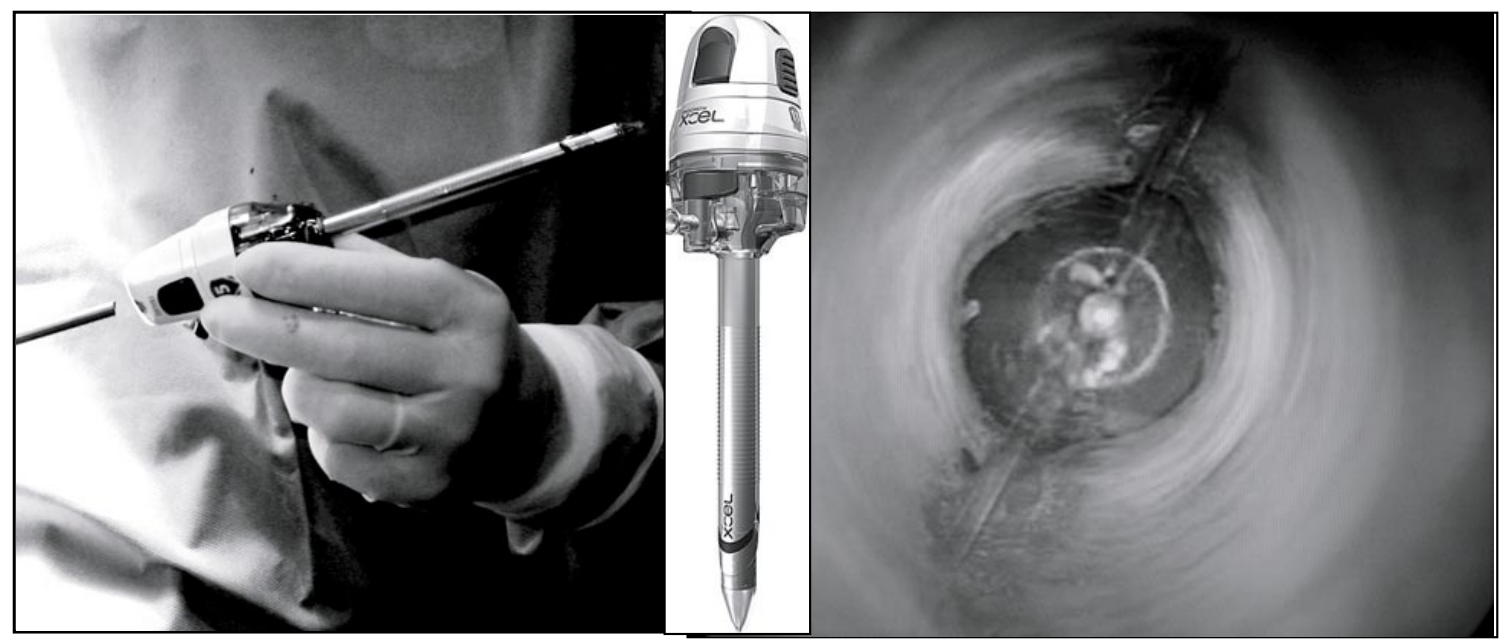

Figura 1. Posición de la cámara al interior del trocar Xcel. En el recuadro central se muestra el detalle del trocar utilizado. Visión durante la entrada en pared abdominal. A través del trócar se observa el útero.

Una vez ingresados en la cavidad abdominal, se observó la ausencia de líquido libre. En relación a anexo izquierdo, se identificó un quiste paratubario, con un diámetro mayor de $6 \mathrm{~cm}$ (Figura 2). Se encontraba el anexo torcido, con dos vueltas sobre el eje de la trompa. Tanto el ovario como la trompa no mostraban signos de isquemia. Se revierte la torsión, dando vueltas en el eje, en sentido contrario. Se realiza una quistectomía. Primero, se diseca parcialmente el quiste con tijera, completando el procedimiento por medio de hidrodisección con suero a presión con bomba (Figura 3 y 4). Luego se aspira el contenido y finalmente se extrae por uno de los trocares (Figura 5 y 6 ). Se revisa la hemostasia y la indemnidad de la trompa y del ovario izquierdo. Luego se revisa el anexo contralateral y el resto de la cavidad pélvica y abdominal. Se extraen los trocares y se sutura sólo la piel.

La paciente evoluciona satisfactoriamente, destacando ausencia de dolor desde el post operatorio inmediato. Se da el alta hospitalaria a las 48 horas, sin complicaciones obstétricas, continuando su gestación en forma fisiológica. No se utilizaron tocolíticos, requiriendo sólo analgesia mínima. Se realimentó a las 6 horas y reinició la deambulación a las 8 horas.

El estudio histológico de anatomía patológica informó que se trataba de un quiste seroso paratubario.

\section{DISCUSIÓN}

Ante la necesidad de una cirugía abdominal durante el embarazo, debemos intentar realizar aquel procedimiento quirúrgico que sea lo más inocuo,

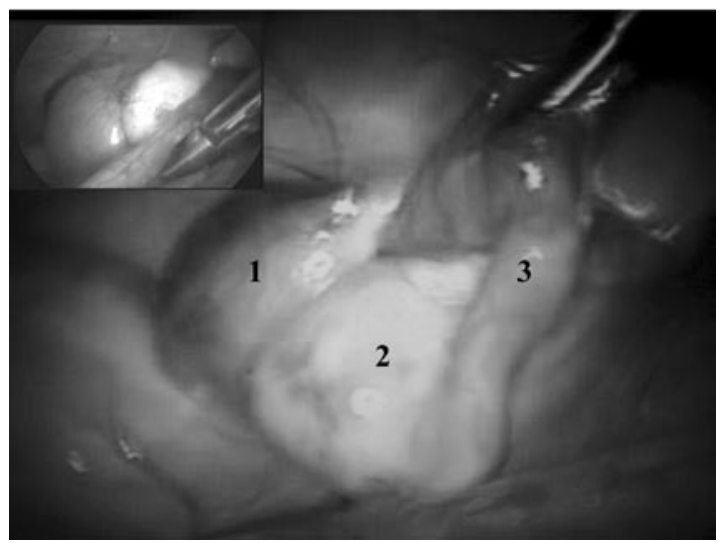

Figura 2. Visión del quiste paratubario (1), ovario (2) y trompa (3). Se observa la torsión de la trompa y quiste.

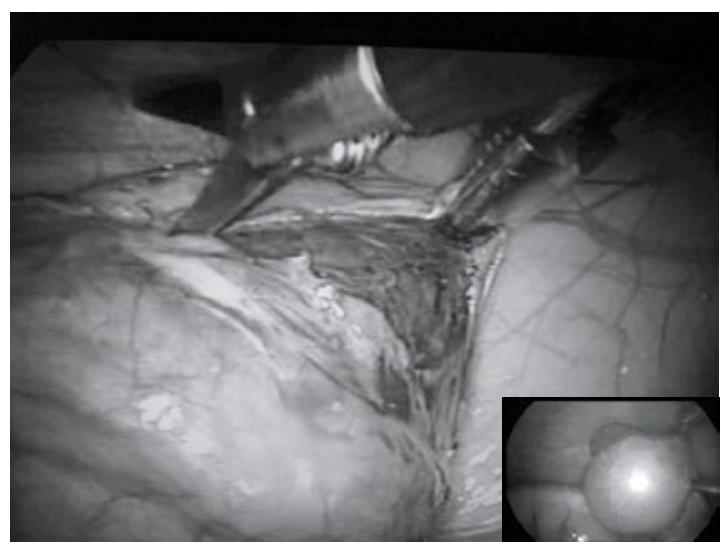

Figura 3. Apertura con tijera de la serosa del quiste paratubario. En el recuadro inferior, se observa el quiste completo. 


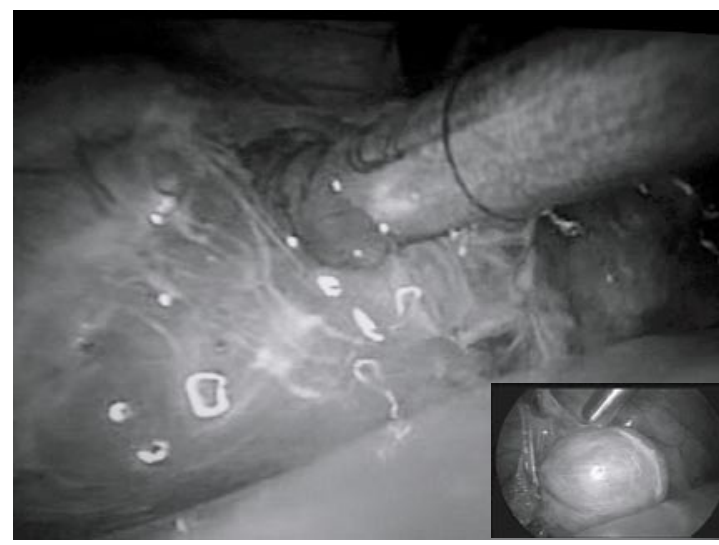

Figura 4. Hidrodisección. Una vez abierta la serosa del quiste, se introduce el irrigador, realizando la separación del quiste, a través de suero a presión. En recuadro se observa el quiste separado casi en su totalidad.

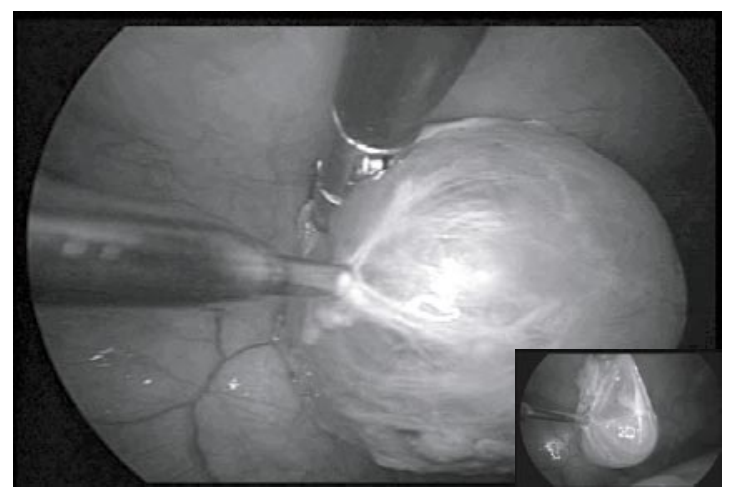

Figura 5. Quiste completo una vez disecado. Se punciona para extraer el contenido líquido.

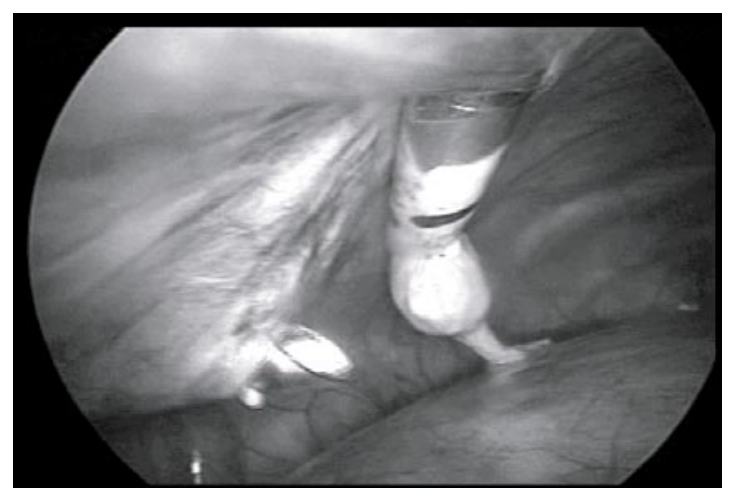

Figura 6. Extracción del quiste a través del trocar.

de tal forma de no alterar el curso del embarazo. La laparoscopia ha demostrado ser menos invasiva que las cirugías por laparotomía. Esto también es aplicable para la embarazada. Por lo tanto, esta vía de abordaje nos permitirá desarrollar todas las ventajas de la mínima invasión, evitando grandes incisiones abdominales, que reducen la capacidad respiratoria en el post operatorio. Por otro lado, debemos recordar que al realizar una incisión en la pared abdominal de una paciente embarazada, al transcurrir el tiempo, con el crecimiento uterino, aumentará la presión sobre la cicatriz y existirá mayor probabilidad de una hernia incisional. Este riesgo disminuye al realizar cirugía laparoscópica, con incisiones mínimas. La recuperación de una laparoscopia es más rápida, con menos dolor, reduciendo la estadía hospitalaria; tal como ocurrió en este caso. Al reducir el dolor, también disminuye la necesidad de medicamentos, como analgésicos en el post operatorio. Además, permite una deambulación y alimentación precoz (Tabla I).

\section{Tabla I}

VENTAJAS DE LA CIRUGÍA LAPAROSCÓPICA EN EL EMBARAZO

1. No requiere grandes incisiones en la pared abdominal

2. Reduce la estadía hospitalaria

3. Recuperación más rápida, deambulación y alimentación precoz.

4. Menor requerimientos de analgesia post-operatoria, al tener menos dolor al compararla con la laparotomía

5. Menor posibilidad de afectar la capacidad respiratoria, al haber menos dolor y una reduccion de la cicatrizen la pared abdominal

Es recomendable trabajar con la menor presión de $\mathrm{CO} 2$ que permita obtener un adecuado neumoperitoneo, para así lograr una distensión de la cavidad abdominal y un campo operatorio satisfactorio. Indudablemente que no podemos esperar un gran campo quirúrgico, ya que el espacio es ocupado por el útero gravídico, lo que reduce significativamente la maniobrabilidad operatoria. Por otro lado, el aumento de la visión en profundidad, por medio de la cámara, es una ventaja comparativa versus la laparotomía. En esta última, probablemente no obtendremos una visión de esta calidad y con tal grado de iluminación. Esto puede ser otra ventaja para el cirujano, si lo comparamos con la cirugía por laparotomía.

En este caso, utilizamos una presión de 15 $\mathrm{mmHg}$, tal como ha sido descrita en otros trabajos, donde se ha comprobado que no aumentan los riesgos tanto para la madre como para el feto, al 
emplear presiones entre 12 y $15 \mathrm{mmHg}(17,18)$. Experimentos en que se midieron los cambios hemodinámicos en ovejas preñadas, demostraron que no hay variaciones significativas en el flujo materno útero-placentario con presiones menores o iguales a $13 \mathrm{~mm} \mathrm{Hg} \mathrm{(19).}$

Teóricamente, el $\mathrm{CO} 2$ del neumoperitoneo podría absorberse por el peritoneo, produciendo una acidosis materno-fetal. Modelos experimentales en ovejas preñadas, han demostrado que presiones menores o iguales a $15 \mathrm{mmHg}$, podrían llevar a una acidosis fetal de corta duración, que no produce efectos dañinos a largo plazo (20). Por lo anterior, como precaución debemos reducir el tiempo operatorio y la presión de $\mathrm{CO} 2$ durante la laparoscopia en la embarazada. Se sabe que los cambios fisiológicos del embarazo, teóricamente, podrían exponer a un mayor riesgo de hipercapnia e hipoxemia a la paciente embarazada frente al neumoperitoneo (18). Sin embargo, si se toman todas las medidas de monitoreo, con la misma rigurosidad que en una mujer no embarazada, se ha comprobado que no se generan mayores problemas (21).

Hoy existen un gran número de recursos y herramientas en laparoscopia, que nos permiten hacer aún menos invasiva la cirugía. Tal es el caso de utilizar trocares como el Xcel, que otorga la ventaja de disminuir la probabilidad de daño al ingreso, por punción del útero. Estos trocares permiten la entrada bajo visión directa, disminuyendo una de las mayores complicaciones durante el acceso a la cavidad abdominal, como es la perforación de vísceras (22), que en el caso de la embarazada corresponde al útero. Este trocar permite la visión directa de los planos al acoplar la cámara en su interior. De esta forma, se puede apreciar la presencia de vasos sanguíneos de la pared, evitando su ruptura. Una vez ingresado a la cavidad peritoneal, visualizaremos el útero, deteniendo la maniobra de inserción, para evitar un potencial daño de la pared uterina.

Las características de este trocar, a diferencia de los de punta piramidal y particularmente los metálicos, es que posee una punta que tracciona, separa y divulsiona, por lo que si hay contacto con la pared uterina, es menos probable un daño. Además, al separar la aponeurosis, no requiere sutura de la misma, una vez retirado el instrumental, sin aumentar la posibilidad de hernias a futuro. Por otra parte, se reduce el tiempo operatorio, disminuyendo la exposición materno-fetal a la anestesia.

En este caso, el útero se encontraba bajo la zona umbilical, lo que nos permitió la inserción de la aguja de Veress a este nivel. Sin embargo, si por el crecimiento uterino, se ve impedida la entrada por esta zona, debemos recordar que existen al- ternativas, como son la línea medio clavicular en el cuadrante superior izquierdo abdominal y la línea medio clavicular en el cuadrante superior derecho abdominal.

En resumen, los riesgos potenciales de una laparoscopia durante el embarazo, están dados básicamente por posibles lesiones de entrada con los trocares, a nivel de vísceras, particularmente el útero y las complicaciones de una cirugía prolongada con presiones aumentadas de $\mathrm{CO} 2$. Todos estos riesgos son evitables, como hemos analizado en los puntos anteriores (Tabla II). Realizar hoy en día una cirugía mínimamente invasiva durante el embarazo, especialmente en el segundo trimestre, tomando todas las medidas ya discutidas, no constituye un mayor riesgo para la madre ni para el feto.

\section{Tabla II}

\section{RIESGOS POTENCIALES DE LA LAPAROSCO- PIA DURANTE EL EMBARAZO Y LAS MEDIDAS QUE PODEMOS REALIZAR PARA REDUCIR SU PROBABILIDAD DE APARICIÓN}

1. Daño de la pared uterina durante la entrada con los trocares.

Utilización de trocares que permitan una entrada bajo visión directa.

Evitar el uso de trocares con puntas de metal o muy aguzadas, que facilitan el daño sobre la pared uterina, en caso de que inadvertidamente se realica presión sobre el útero durante la entrada.

Evitar la entrada umbilical en caso de úteros que se encuentren bajo esta posición, debido a la edad gestacional mayor. Utilización de las entradas alternativas, que son para cualquier laparoscopia.

2. Hipoxia fetal por disminución del flujo uterino, por neumoperitoneo con una presión de $\mathrm{CO} 2$ alta.

Reducción del tiempo operatorio y de la presión de $\mathrm{CO} 2$

3. Acidosis fetal por absorción de CO2 del neumoperitoneo.

Reducción del tiempo operatorio y de la presión de $\mathrm{CO} 2$.

Si bien, en la gran mayoría de los casos se indica una cirugía, cuando la clínica demuestra que se trata de una masa anexial complicada, una apendicitis o colecistitis aguda, no debemos esperar la alteración extrema de los parámetros sanguíneos, que sólo son el reflejo de un mayor componente inflamatorio y por ende un mayor compromiso materno. Al diferir la cirugía, aumentan las probabilidades de una pérdida fetal o un mayor compromiso materno. En la literatura se reportan series de apen- 
dicitis operadas tempranamente con hasta 1,5\% de pérdidas fetales, mientras que en las operadas tardíamente, la cifra se incrementa hasta un 35\% (23). Indudablemente, que el aumento de sustancias inflamatorias, como las prostaglandinas, pueden contribuir a desencadenar la serie de eventos que culminará en una pérdida fetal. Esto también es aplicable en masas anexiales torcidas. Por otro lado, el síntoma dolor, generalmente se presenta encubierto o atenuado durante la gestación, lo que debemos tener presente para realizar un diagnóstico precoz frente a patología con abdomen agudo en el embarazo.

\section{CONCLUSIONES}

La resolución laparoscópica de tumores anexiales complicados, durante el segundo trimestre del embarazo, según nuestra experiencia es segura y eficaz. Si la comparamos con la laparotomía, permite realizar una cirugía mínimamente invasiva, reduciendo la estadía hospitalaria con una rápida recuperación. Disminuye la posibilidad de complicaciones maternas y fetales. No requiere el uso de tocolíticos de rutina. El avance en la tecnología, permite disponer de herramientas que hacen aún menos invasiva, una técnica que ya lo es. La entrada bajo visión directa con cámara, permite reducir la posibilidad de daño uterino, durante el acceso a la cavidad abdominal.

\section{BIBLIOGRAFÍA}

1. Malinowski A, Szpakowski M, Kolasa D, Wieczorek A, Nowak M, Wilczyński JR, Szyłło K. Laparoscopy in pregnant women. Ginekol Pol 2002;73(4):247-54.

2. Mathevet P, Nessah K, Dargent D, Mellier G. Laparoscopic management of adnexal masses in pregnancy: a case series. Eur J Obstet Gynecol Reprod Biol 2003;108(2):217-22.

3. Larraín D, Durruty G, Pomés C, Cuello M. Consideraciones para el uso de la laparoscopia durante el embarazo. Rev Chil Obstet Ginecol 2007;72(4):247-57

4. Argenta PA, Nezhat F. Approaching the adnexal mass in the new millennium. J Am Assoc Gynecol Laparosc 2000;7(4):455-71.

5. Steiner RA, Fehr PM. Minimal invasive surgery in gynaecology. Ther Umsch 2005;62(2):127-38.

6. Moreno-Sanz C, Pascual-Pedreño A, Picazo-Yeste JS, Seoane-Gonzalez JB. Laparoscopic appendectomy during pregnancy: between personal experiences and scientific evidence. J Am Coll Surg 2007;205(1):3742.

7. Popkharitov A, Lazarov L, Popkharitov I, lovchev I,
Matev A, Lazarov N. Laparoscopic cholecystectomy during pregnancy. Case report and literature review. Akush Ginekol (Sofiia) 2007;46(1):49-52.

8. Nezhat FR, Tazuke S, Nezhat CH, Seidman DS, PhiIlips DR, Nezhat CR. Laparoscopy during pregnancy: a literature review. JSLS 1997;1(1):17-27.

9. Moore RD, Smith WG. Laparoscopic management of adnexal masses in pregnant women. J Reprod Med 1999;44(2):97-100.

10. Curet MJ, Allen D, Josloff RK, Pitcher DE, Curet LB, Miscall BG, Zucker KA. Laparoscopy during pregnancy. Arch Surg 1996;131(5):546-50.

11. El-Yahia AR, Rahman J, Rahman MS, al-Suleiman SA. Ovarian tumours in pregnancy. Aust N Z J Obstet Gynaecol 1991;31(4):327-30.

12. Leiserowitz GS, Xing G, Cress R, Brahmbhatt B, Dalrymple JL, Smith LH. Adnexal masses in pregnancy: how often are they malignant? Gynecol Oncol 2006;101(2):315-21.

13. Nowak M, Szpakowski M, Wilczyński JR. Ovarian tumors in pregnancy--proposals of diagnosis and treatment. Ginekol Pol 2004;75(3):242-9.

14. Whitecar MP, Turner S, Higby MK. Adnexal masses in pregnancy: a review of 130 cases undergoing surgical management. Am J Obstet Gynecol 1999;181(1):1924.

15. Chittacharoen A, Wangpusayavisut A, O-Prasertsawat $P$. Adnexal masses in pregnancy. J Med Assoc Thai 2005;88 Suppl 2:S37-40.

16. Kumari I, Kaur S, Mohan H, Huria A. Adnexal masses in pregnancy: a 5-year review. Aust N Z J Obstet Gynaecol 2006;46(1):52-4.

17. Lenglet $Y$, Roman $H$, Rabishong $B$, Bourdel N, Bonnin M, Bolandard F, Duband P, Pouly JL, Mage G, Canis M. Laparoscopic management of ovarian cysts during pregnancy. Gynecol Obstet Fertil 2006;34(2):101-6.

18. O'Rourke N, Kodali BS. Laparoscopic surgery during pregnancy. Curr Opin Anaesthesiol 2006;19(3):2549.

19. Barnard JM, Chaffin D, Droste S, Tierney A, Phernetton $\mathrm{T}$. Fetal response to carbon dioxide pneumoperitoneum in the pregnant ewe. Obstet Gynecol 1995;85(5 Pt 1):669-74.

20. Curet MJ, Vogt DA, Schob O, Qualls C, Izquierdo LA, Zucker KA. Effects of CO2 pneumoperitoneum in pregnant ewes. J Surg Res 1996;63(1):339-44.

21. Colomb S, Bonnin M, Bolandard F, Lenglet $Y$, Duband $\mathrm{P}$, Roman $\mathrm{H}$, Canis $\mathrm{M}$, Bazin JE. Pregnant woman anaesthetic management in gynaecologic laparoscopic surgery at the maternity hospital of Clermont-Ferrand. Ann Fr Anesth Reanim 2006;25(1):11-6.

22. Vilos GA, Ternamian A, Dempster J, Laberge PY. Laparoscopic entry: a review of techniques, technologies, and complications. J Obstet Gynaecol Can 2007;29(5):433-65.

23. Al-Fozan H, Tulandi T. Safety and risks of laparoscopy in pregnancy. Curr Opin Obstet Gynecol 2002;14(4):375-9. 